\title{
Photonic billiards in ultra-peripheral heavy-ion collisions
}

Mariola Kłusek-Gawenda*

Institute of Nuclear Physics Polish Academy of Sciences, PL-31342 Krakow, Poland

E-mail: mariola.klusek@ifj.edu.pl

Ultra-peripheral heavy ion collisions are a source of photons which collide with the other nucleus producting single vector meson or they can collide with each other producing a pair of particles. The contribution from fermionic boxes, resonance scattering, VDM-Regge model, two-gluon exchange and pionic background will be studied to two-photon production. Each of these processes dominates at different range of two-photon invariant masses. Our results for nuclear cross section are in good agreement with recently measured ATLAS and CMS data. Predictions including ALICE and LHCb semi(acceptance) for the next run at the LHC will be shown.

European Physical Society Conference on High Energy Physics - EPS-HEP2019 -

10-17 July, 2019

Ghent, Belgium

${ }^{*}$ Speaker. 


\section{Introduction}

Physics of the ultra-peripheral collisions (UPC) of heavy ions gives a nice opportunity to study electromagnetic processes [1, 2, 3, 4]. Due to the very strong electromagnetic field of colliding nuclei, reactions relate to photon collisions can be studied. One can consider $\gamma \gamma$ fusion and photoproduction (Pomeron and/or Reggeon exchange) as a sub-process of heavy ion UPC. This study will concern the light-by-light scattering. Diphoton processes have long been studied at $e^{+} e^{-}$collider. This tool allows to test a QED theory and a lot of aspects of meson spectroscopy. The first theory concerning the possibility of the light-by-light scattering was proposed more than 80 years ago i.a. by W. Heisenberg and his students : H. Euler and B. Kockel [5, 6] or by A. Akhieser, L. Landau and I. Pomeranchuk [7].

\section{Methodology}

(c)

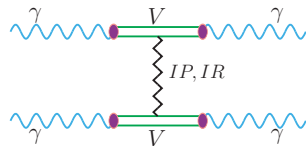

(a)

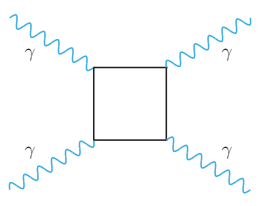

(b)

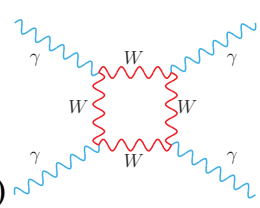

(e)

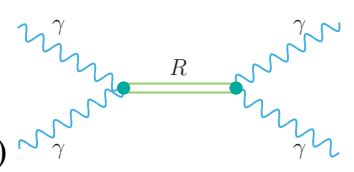

Figure 1: $\gamma \gamma \rightarrow \gamma \gamma$ scattering: (a) fermionic boxes, (b) one-loop $W$ box, (c) the VDM-Regge, (d) two-gluon exchange mechanism and (e) resonance scattering.

The leading order of elementary cross section for $\gamma \gamma \rightarrow \gamma \gamma$ process is well-know and one can use an available to the general public Mathematica package: FormCalc [8]. The (a) diagram in Fig. 1 shows so-called fermionic box (scattering via quarks and leptons is taken into account). The next diagram presents $W^{+} W^{-}$boson loop and this cross section is calculated within LoopTools [9]. Fig. 1(c) presents diagram for non-perturbative mechanism of both photons fluctuation into vector mesons and their subsequent interaction. This involves the Reggeon and Pomeron exchanges between $\rho, \omega$ or $\phi$ light mesons. The (d) diagram of Fig. 1 is the same order in $\alpha_{e m}$ as previous one but has higher order in $\alpha_{s}$. The two-gluon exchange mechanism is a three-loop mechanism [10]. The finite fermion masses, the full momentum structure in the loops and all helicity amplitudes are included. Considering processes with gluon exchange, one can use so-called regularization parameter, $m_{g}$, which can be: $m_{g}=0$ for usual gluon exchange, however $m_{g}=0.75 \mathrm{GeV}$ is suggested by lattice QCD [11]. In the present analysis we take into account pseudoscalar and scalar mesons that decay into two-photons: $\eta, \eta^{\prime}(958), \eta_{c}(1 S), \eta_{c}(2 S), \chi_{c 0}(1 P)$. The amplitude for the $\gamma \gamma$ production through the $s$-channel exchange of a pseudoscalar/scalar mesons is the same as in Ref. [12]. In addition, also background from the $\gamma \gamma \rightarrow \pi^{0}(\rightarrow \gamma \gamma) \pi^{0}(\rightarrow \gamma \gamma)$ process is considered. In Ref. [12] we constructed a multi-component model which describes e.g. the Belle [13] and Crystal Ball [14] data for $\gamma \gamma \rightarrow \pi^{0} \pi^{0}$. Both $\gamma \gamma \rightarrow \pi^{+} \pi^{-}$and $\gamma \gamma \rightarrow \pi^{0} \pi^{0}$ reactions were considered within a multi-component model in Ref. [12]. There, for the first time, both the total cross section and 
angular distributions and significance of nine resonances, $\gamma \gamma \rightarrow \pi^{+} \pi^{-} \rightarrow \rho^{ \pm} \rightarrow \pi^{0} \pi^{0}$ continuum, Brodsky-Lepage and handbag mechanisms in these processes was studied. A detailed formalism and the description of these sub-processes can be found in [12]. If only two photons from different neutral pions are measured at a given experimental range of rapidities and transverse momenta such an event could be wrongly identified as $\gamma \gamma \rightarrow \gamma \gamma$ scattering if no extra cuts are imposed to reduce or eliminate such a background.

The nuclear cross section is calculated with the help of equivalent photon approximation (EPA) $[15,16,17]$. This theory uses concept of photon fluxes which depend on energy of photon $\left(\omega_{i}\right)$ and impact parameter $\left(\mathbf{b}_{i}\right)$. Here we consider only ultra-peripheral collisions that is impact parameter (b) determining distance between medium of colliding nuclei is bigger than the sum of the radii. The end of $\mathbf{b}_{i}$ vectors set a position where the pair of particles is produced (two-photon production). To calculate nuclear cross section for $A A \rightarrow A A X_{1} X_{2}$ process one needs to know the probability that first nucleus emits a photon with the energy $\omega_{1}$ and, simultaneously, second nucleus emits a photon with the energy $\omega_{2}$. Next, they collide with each other to produce some final state $X_{1} X_{2}$ (here it is $\gamma \gamma$ or $\pi^{0} \pi^{0}$ state). In our approach, we use equivalent photon approximation in the impact parameter space. The total cross section takes the form of five-fold integral:

$$
\begin{aligned}
\sigma_{A_{1} A_{2} \rightarrow A_{1} A_{2} X_{1} X_{2}}\left(\sqrt{s_{A_{1} A_{2}}}\right) & =\int \sigma_{\gamma \gamma \rightarrow X_{1} X_{2}}\left(W_{\gamma \gamma}\right) N\left(\omega_{1}, \mathbf{b}_{\mathbf{1}}\right) N\left(\omega_{2}, \mathbf{b}_{2}\right) S_{a b s}^{2}(\mathbf{b}) \\
& \times \mathrm{d}^{2} b \mathrm{~d} \bar{b}_{x} \mathrm{~d} \bar{b}_{y} \frac{W_{\gamma \gamma}}{2} \mathrm{~d} W_{\gamma \gamma} \mathrm{d} Y_{X_{1} X_{2}},
\end{aligned}
$$

where $\bar{b}_{x}$ and $\bar{b}_{y}$ are components of $\mathbf{b}_{i}$ vector, the energy of produced particles depends on energy of photons as follows: $W_{X_{1} X_{2}}=2 \sqrt{\omega_{1} \omega_{2}}$ and rapidity of outgoing system is expressed through rapidity of singles particles: $Y_{X_{1} X_{2}}=\frac{1}{2}\left(y_{X_{1}}+y_{X_{2}}\right)$. Absorption factor $S_{a b s}(\mathbf{b})$ assures UPC which means that the nuclei do not undergo nuclear breakup. Very often we extend Eq. 2.1 by additional dimension. This allows to make a more accurate prediction or description of experimental data.

The flux of photons, $N(\omega, b)$, very strongly depends on the nuclear form factor at small $b$. We prefer to use a realistic form factor in our calculation. Then nucleus is treated as a object with realistic charges distribution. Thereby the Wood-Saxon density profile is included. Often a monopole form factor is used in the calculation. The comparison of results that include realistic and monopole form factor will be presented below. Nuclear form factor kills large virtualities in UPC of heavy ion, therefore, the initial photon virtualities equal to almost zero.

A current analysis concerns the production of diphoton pairs originating from boxes, VDMRegge mechanism, resonances which decay into $\gamma \gamma$ state and pionic background. Eq. (2.1) enables to calculate nuclear cross section for each of above processes.

\section{Theoretical results}

Light-by-light scattering was realized experimentally only recently $[18,19,20]$. Simultaneously, we have studied light-by-light scattering via fermion loop and with the help of nonperturbative mechanism of photons fluctuation into light vector mesons (VDM-Regge model) [21]. We have considered only ultra-peripheral lead-lead collisions. For ions of charges $Z_{1}, Z_{2}$, the cross section is enhanced by $Z_{1}^{2} Z_{2}^{2}$ factor compared to proton-proton collisions, at least at low diphoton invariant masses equal to diphoton collision energies, where the initial photons are quasi real 
with extremely low virtualities. But on the other hand, a significant part of cross section is cut by absorption factor (Eq. (2.1)) which ensures ultra-peripheral character of the process.

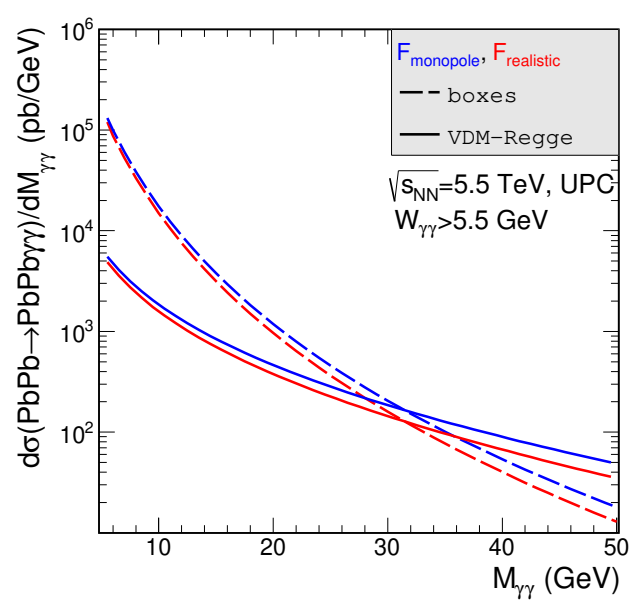

Figure 2: Differential cross section as a function of diphoton invariant mass. Blue lines - nuclear calculations include the monopole form factor. Red lines - results with the realistic form factor. Dashed lines - boxes contribution and solid lines relates to VDM-Regge model.

Fig. 2 illustrates a comparison of contributions of boxes (dashed lines) and VMD-Regge mechanism (solid lines) in nuclear calculations taking into account the monopole (blue lines) and realistic form factor (red lines). One can see a differential cross section for the $\mathrm{PbPb} \rightarrow \mathrm{PbPb} \gamma \gamma$ reaction in heavy ion UPC at $\sqrt{s_{N N}}=5.5 \mathrm{TeV}$ with extra cut on $W_{\gamma \gamma}>5.5 \mathrm{GeV}$. The cross section obtained with the monopole form factor is more than $10 \%$ bigger than that obtained with the form factor which is calculated as a Fourier transform of the charge distribution in the nucleus. The ratio between those two results become larger with larger value of the diphoton invariant mass. The competition of the two mechanisms seems to be interesting. While at low energies the box contribution wins, at $M_{\gamma \gamma}>30 \mathrm{GeV}$ the VDM-Regge contribution is bigger.

ATLAS measured a fiducial cross section of $\sigma=78 \pm 13$ (stat.) \pm 7 (syst.) \pm 3 (lumi.) nb $[18,19]$ and our theoretical calculations (including experimental acceptance) gave $51 \pm 0.02 \mathrm{nb}$ [21]. The ATLAS comparison of its experimental results to the predictions from Ref. [21] shows a reasonable agreement (see Fig. 3 in Ref. [18]). The ATLAS detector recorded data of lead-lead collision at the centre-of-mass energy per nucleon pair of $5.02 \mathrm{TeV}$. Measurement of diphoton pair was done in midrapidity region. The $\gamma \gamma$ invariant mass was limited to $M_{\gamma \gamma}>6 \mathrm{GeV}$. Similarly as the ATLAS collaboration, the CMS group measured the same process but for somewhat lower threshold of invariant mass of the produced diphotons [20]. The measured fiducial light-by-light scattering cross section, $\sigma=120 \pm 46$ (stat.) \pm 28 (syst.) \pm 4 (theo.) nb. We have recalculated this process including the CMS acceptance and we obtained $\sigma=(103 \pm 0.034) \mathrm{nb}$ which is in a good agreement with data.

Due to relatively large cuts on the photon transverse momenta, only relatively large diphoton invariant masses were measured by the ATLAS and CMS collaborations. We believe that in a future one could go to larger luminosity, higher collision energies, better statistics and smaller diphoton invariant masses. 
Next, predictions for the ALICE and LHCb experiment will be shown. The ALICE detector facilitates a measurement of outgoing photons at midrapidity region $-0.9<\eta_{\gamma}<0.9$ [22]. Photons with transverse energy smaller than $200 \mathrm{MeV}$ cannot be detected. Also predictions that include $\mathrm{LHCb}$ (semi)acceptance will be presented. Then more forward rapidity region $2<\eta_{\gamma}<4.5$ [23] is taken into account. Here we assume that any photon with $p_{t, \gamma}>200 \mathrm{MeV}$ will be measured.

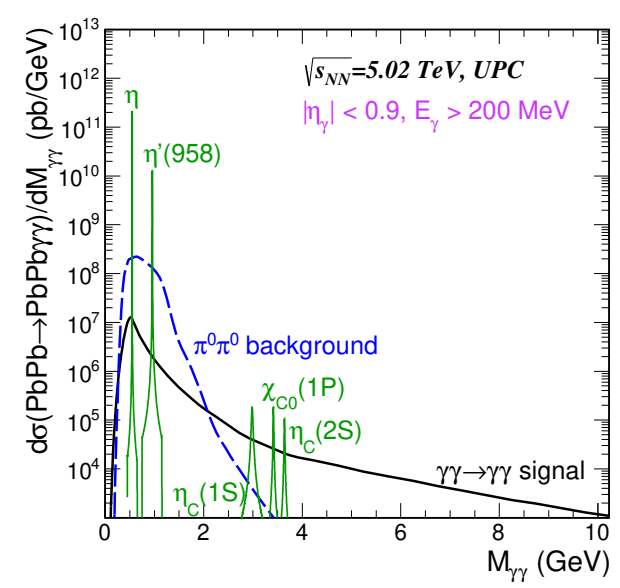

(a)

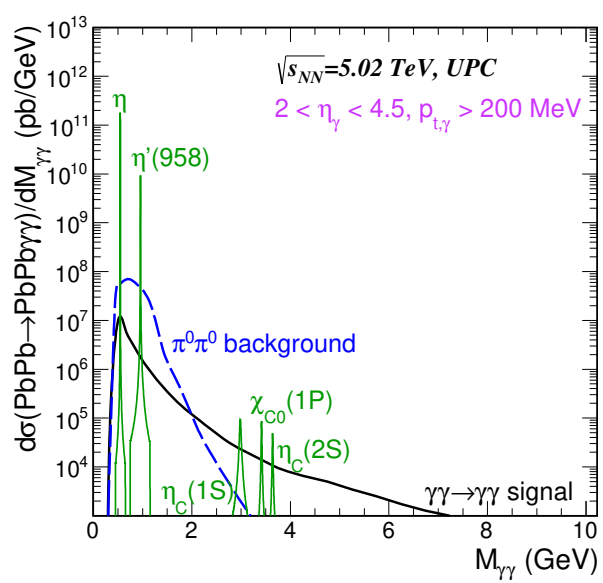

(b)

Figure 3: Differential nuclear cross section for $\mathrm{PbPb} \rightarrow \mathrm{PbPb \gamma \gamma}$ as a function of $\gamma \gamma$ invariant mass. (a) ALICE and (b) LHCb kinematical cuts are included. In addition, the energy experimental resolution is taken into account. Here $\sqrt{s_{N N}}=5.02 \mathrm{TeV}$.

Fig. 3 illustrates contribution of $\gamma \gamma \rightarrow \gamma \gamma$ signal (black line), background (blue dashed line) and resonances (green lines) to the diphoton invariant mass distribution for ALICE (a) and LHCb conditions (b). The background is composed of events where exactly two of four outgoing photons are detected. The first one comes from the first pion, and the second one comes from the second pion. The two other photons, from the $\pi^{0} \pi^{0} \rightarrow(\gamma \gamma)(\gamma \gamma)$ decays, are then outside of detection area. Contributions suggest that one could be able to measure the LO QED fermionic signal above $M_{\gamma \gamma}>2 \mathrm{GeV}$. Below this value, two very clear peaks show up, corresponding to $\eta$ and $\eta^{\prime}(958)$ mesons that decay into two-photon final state. Inclusion of the Gaussian distribution to simulate experimental energy resolution causes only a little smearing of the resonant signals. Then the transverse momenta of each of the photons takes the form: $p_{i, t}=p_{t}+\left(\frac{p_{t}}{E_{i}}\right) \delta E_{i}$. It seems to worth mentioning that the peak corresponding to a resonance very strongly depends on the number of bins. The maximum value of the differential cross section emerges exactly at $m_{R}$.

Fig. 4 corresponds to the next run at the LHC [24]. The energy (per nucleon) of heavyion collision is expressed through the energy in the center-of-mass of proton-proton collision $\sqrt{s_{p p}}=14 \mathrm{TeV}: \sqrt{s_{N N}}=\sqrt{\frac{Z_{1} Z_{2}}{A_{1} A_{2}}} \sqrt{s_{p p}}$. Then predicted value for ${ }^{208} \mathrm{~Pb}^{82+}{ }^{208} \mathrm{~Pb}^{82+}$ collision is $\sqrt{s_{N N}}=5.52 \mathrm{TeV}$ (Fig. 4 (a)) and for ${ }^{40} \mathrm{Ar}^{18+}{ }_{-}^{40} \mathrm{Ar}^{18+}$ it is $\sqrt{s_{N N}}=6.3 \mathrm{TeV}$ (Fig. 4 (b)). The analysis focuses on lower diphoton invariant masses again. At lower energies $\left(W_{\gamma \gamma}<4 \mathrm{GeV}\right)$ meson resonances may play important role in addition to the Standard Model box diagrams or proposed pionic background. The inclusion of energy resolution has a significance mainly at $\gamma \gamma \rightarrow \eta, \eta^{\prime} \rightarrow \gamma \gamma$ resonance scattering and this contribution will be measured with a good statistics. However, the resonance signal is modified including experimental energy resolution and the $\eta$ and $\eta^{\prime}(958)$ peaks 


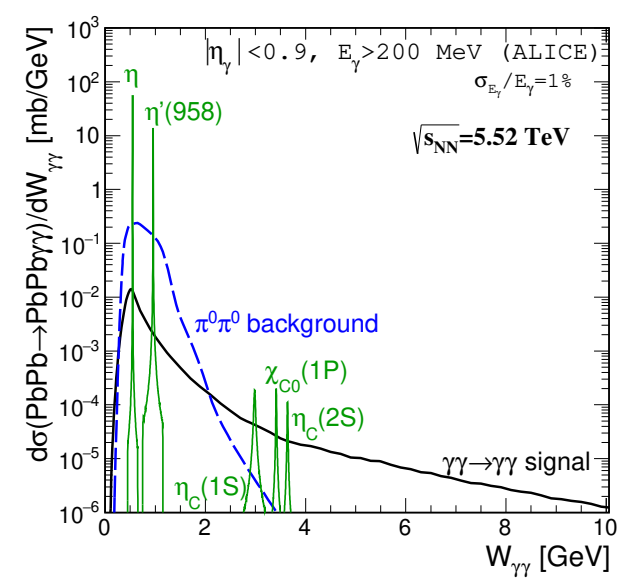

(a)

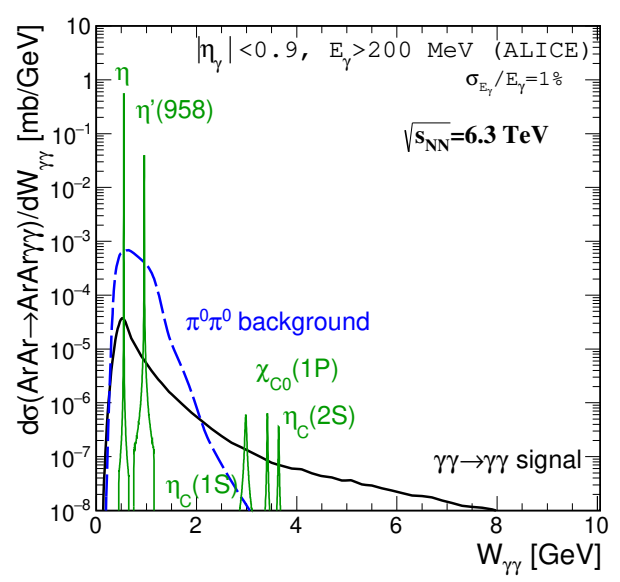

(b)

Figure 4: Differential cross section as a function of $W_{\gamma \gamma}=M_{\gamma \gamma}$ for (a) $\mathrm{PbPb} \rightarrow \mathrm{PbPb} \gamma \gamma$ and (b) $\operatorname{ArAr} \rightarrow \operatorname{ArAr} \gamma \gamma$. The collision energy at the center-of-mass of the heavy ion collision is $5.52 \mathrm{TeV}$ and 6.3 TeV for lead-lead and argon-argon respectively.

are about one order of magnitude smaller than without experimental resolution but the total cross section is of course still the same. These figures suggest that one could be able to measure the $\gamma \gamma \rightarrow \gamma \gamma$ scattering above $W_{\gamma \gamma}>2 \mathrm{GeV}$. Comparing Fig. 4 (a) with (b) one can observe that the relevant distribution varies more than two orders of magnitude. In the case of argon-argon collisions, although the collision energy is larger, the predicted cross section is smaller. This is caused by fourth power of the charge number of the nucleus in the cross section. The photon flux depends on $Z_{A}^{2}$ so the cross section is multiplied by $Z_{A}^{4}$. Thus the total cross section for lead-lead collision is more than two orders of magnitude larger than for the argon-argon collision case.

\section{Conclusion}

When photons collide with each other, they act like billiard balls, both spring away from each other. The ultra-peripheral heavy ion collisions give a possibility to measure the $\gamma \gamma \rightarrow \gamma \gamma$ scattering. So far the ATLAS and CMS collaborations measured the light-by-light scattering for diphoton collisions energies $W_{\gamma \gamma}>6 \mathrm{GeV}$ (ATLAS) and $W_{\gamma \gamma}>5 \mathrm{GeV}$ (CMS). Calculated by us the Standard Model predictions are roughly consistent with the experimental data. Our results include realistic photon fluxes that is a Fourier transform of the charge distribution in the nucleus. We have proposed several additional mechanisms which contribute to two-photon state. Each of them plays a important role and different ranges of diphoton invariant mass or transverse momentum of single photon [25]. The effect of energy resolution has a major impact on smearing of mesonic contribution and does not change the value of the cross section. The largest cross section is obtained for the $\gamma \gamma \rightarrow \eta \rightarrow \gamma \gamma$ resonance scattering. Additionally, in the range of diphoton invariant mass $M_{\gamma \gamma}>2 \mathrm{GeV}$, comparison of cross sections for fermionic boxes and pionic background clearly shows almost fourfold dominance of boxes over non-wanted background. Comparing contributions for lead-lead and argon-argon collisions, one can deduce that collision of lighter nuclei is less favorable. However, we can hope the luminosity in the run with Ar-Ar collision will be higher. 


\section{References}

[1] V.M. Budnev, I.F. Ginzburg, G.V. Meledin and V.G. Serbo, Phys. Rept. 15, 181 (1975)

[2] M. Kłusek-Gawenda and A. Szczurek, Phys. Rev. C82, 014904 (2010)

[3] A. van Hameren, M. Kłusek-Gawenda and A. Szczurek, Phys. Lett. B776, 84 (2018)

[4] C. Azevedo, V.P. Goncalves and B.D. Moreira, Eur. Phys. J. C79, 432 (2019)

[5] H. Euler and B. Kockel, Naturwiss. 23, 246 (1935)

[6] W. Heisenberg and H. Euler Zeit. f. Phys. 98, 714 (1936)

[7] A. Akhieser, L. Landau and I. Pomeranchuk, Nature 138, 206 (1936)

[8] T. Hahn and M. Perez-Victoria, Comput. Phys. Commun. 118, 153 (1999)

[9] G. J. van Oldenborgh and J. A. M. Vermaseren, Z. Phys. C46, 425 (1990)

[10] M. Kłusek-Gawenda, W. Schäfer and A. Szczurek, Phys. Lett. B761, 399 (2016)

[11] E. Meggiolaro, Phys. Lett. B451, 414 (1999)

[12] M. Kłusek-Gawenda and A. Szczurek, Phys. Rev. C87, 054908 (2013)

[13] Belle Collaboration (S. Uehara et al.), Phys. Rev. D79, 052009 (2009)

[14] Crystal Ball Collaboration (H. Marsiske et al.), Phys. Rev. D41, 3324 (1990)

[15] E. Fermi, Nuovo Cimento 2, 143 (1925)

[16] C. F. Weizsäcker, Z. Phys. 88, 612 (1934)

[17] E. J. Williams, Phys. Rev. 45, 729 (1934)

[18] ATLAS Collaboration (M. Aaboud et al.), Nature Phys. 13, 852 (2017)

[19] ATLAS Collaboration (G. Aad et al.), Phys. Rev. Lett. 123, 052001 (2019)

[20] CMS Collaboration (A. M. Sirunyan et al.), Phys. Lett. B797, 134826 (2019)

[21] M. Kłusek-Gawenda, P. Lebiedowicz and A. Szczurek, Phys. Rev. C93, 044907 (2016)

[22] ALICE Collaboration (B. B. Abelev et al.), Int. J. Mod. Phys. A29, 1430044 (2014)

[23] LHCb Collaboration (M. Clemencic et al.), J. Phys. Conf. Ser. 331, 032023 (2011)

[24] Z. Citron et al., arXiv:1812.06772 [hep-ph]

[25] M. Kłusek-Gawenda, R. McNulty, R. Schicker and A. Szczurek, Phys. Rev. D99, 093013 (2019) 